\title{
Safety, feasibility and efficacy of a rapid ART initiation in pregnancy pilot programme in Cape Town, South Africa
}

\author{
S Black, ${ }^{1}$ MPH; R Zulliger, ${ }^{2}$ MPH; L Myer, ${ }^{1,3}$ MB ChB, PhD; R Marcus, ${ }^{1}$ MB ChB, BSc, DTM\&H; S Jeneker, ${ }^{1}$ BA Hons; \\ R Taliep, ${ }^{4}$ MB ChB, DCH, Dip HIV Man, DTM\&H; D Pienaar, ${ }^{5} \mathrm{MB}$ ChB, FCPHC; R Wood, ${ }^{1,6}$ FCP (SA), DSc (Med); \\ L-G Bekker, ${ }^{1,6} \mathrm{MB}$ ChB, DCH, DTM\&H, FCP (SA), PhD
}

\author{
${ }^{1}$ Desmond Tutu HIV Centre, Institute of Infectious Diseases and Molecular Medicine, University of Cape Town, South Africa \\ ${ }^{2}$ Department of Health, Behavior and Society, John Hopkins Bloomberg School of Public Health, Baltimore, USA \\ ${ }^{3}$ School of Public Health and Family Medicine, University of Cape Town, South Africa \\ ${ }^{4}$ Metro District Health Services, Western Cape (Klipfontein Mitchell's Plain Substructure), Cape Town, South Africa \\ ${ }^{5}$ Provincial Government of the Western Cape, Cape Town, South Africa \\ ${ }^{6}$ Department of Medicine, University of Cape Town, South Africa
}

Corresponding author:S Black (samanthablack19@gmail.com)

\begin{abstract}
Background. Antiretroviral therapy (ART) in pregnancy is a crucial intervention in the prevention of mother-to-child transmission (PMTCT) of HIV. It is recognised that mother-to-child transmission is reduced with each week on ART. However, in most South African settings, ART initiation is delayed owing to slow determination of treatment eligibility and separation of HIV and antenatal care services. Objective. The rapid initiation of an ART in pregnancy programme is a model of care designed to expedite treatment initiation in ARTeligible pregnant women. This study evaluated the performance of the programme.

Methods. Participants enrolled in the ART programme in the same week as their first ANC visit throughout 2011, and had outcome data available by March 2012. Treatment eligibility was determined or confirmed via point-of-care CD4 $4^{+}$testing. Eligible women were offered ART immediately, with concurrent counselling and safety laboratory blood testing. Women attended until 6 - 8 weeks after delivery. Data were collected from clinical records with infant polymerase chain reaction (PCR) results at 6 weeks.

Results. Of 134 ART-eligible (CD4+ count $<350$ cells/ $\mu$ or WHO stage III/IV) pregnant women, 130 (97.0\%) started ART, 118 (90.8\%) initiating treatment the same day that treatment eligibility was determined. There were no abnormal laboratory blood results or adverse events that required medical intervention. Pre-delivery retention in care and infant mortality were comparable to those in similar settings. Of the 107 pregnancies with PCR outcomes available, there was 1 positive HIV result in an infant (0.9\%). Maternal viral load suppression in this mother was not achieved by the time of delivery.

Conclusions. This pilot programme provides evidence that rapid ART initiation in pregnancy is safe, feasible and effective in reducing PMTCT. Further follow-up is required to monitor long-term outcomes.

S Afr Med J 2013;103(8):557-562. DOI:10.7196/SAMJ.6565
\end{abstract}

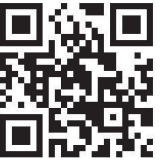

Antiretroviral therapy (ART) is the key strategy in prevention of mother-to-child transmission (PMTCT) of HIV, with co-existing benefits for maternal health. ${ }^{[1]}$ Despite the potential of PMTCT interventions to eliminate paediatric HIV transmission and increased access to ART, in 2010 there were 40000 new HIV paediatric infections in South Africa (SA). ${ }^{[2,3]}$

The most critical determinant of mother-to-child transmission (MTCT) is maternal HIV viral load (VL). ${ }^{[4]}$ ART reduces maternal $\mathrm{VL}$, minimising transmission in utero, during delivery and during the postpartum period. ${ }^{[5,6]}$ The duration of ART necessary for viral suppression at delivery is dependent on the mother's VL at ART commencement. ${ }^{[7]} \mathrm{A}$ recent study highlighted the association between ART duration in pregnancy and MTCT, with vertical transmission rates of $9.3 \%, 5.5 \%$ and $3.5 \%$ documented in women on ART for $<4$ weeks, 4 - 16 weeks and $>16$ weeks before delivery, respectively. ${ }^{\left[{ }^{[8]}\right.}$ In the SA setting, where many women only present to health services in the second to third trimester of pregnancy, ${ }^{[9]}$ expedited initiation may be critical, particularly when HIV is more advanced.

Studies have indicated that suboptimal health system strategies for ART initiation contribute to MTCT. ${ }^{[10]}$ SA currently follows the World Health Organization (WHO) PMTCT Guidelines Option A, with ART-eligible women referred to ART services separate from their antenatal care. This separation often leads to treatment delays and women being lost in the referral process. ${ }^{[11,12]}$ Additionally, determination of treatment eligibility, which includes a $\mathrm{CD} 4^{+}$cell count, the patient preparation procedure, clinical assessment and patient education, can take up to 6 weeks. ${ }^{[13,14]}$ These delays increase the risk of MTCT.

To address these needs, we designed and implemented a pilot programme in Cape Town, SA, with an emphasis on starting women on ART as soon as, and even the same day as, treatment eligibility was determined. This programme, entitled Rapid Initiation in Pregnancy (RAP), sought to strengthen the testing-to-treatment cascade and provide a new approach to treatment readiness. This paper describes the uptake and clinical outcomes, as well as perinatal HIV transmission rates, in a cohort of women who were enrolled in the programme over an 11-month period.

\section{Methods \\ Programme design}

The programme was delivered in a community-based antenatal clinic (ANC) in Cape Town from February 2011 and has been described elsewhere. ${ }^{[15]}$ Briefly, women were tested for HIV at their first ANC 
visit. All HIV-positive women had blood sent for a National Health Laboratory Service (NHLS) panleukogated $\mathrm{CD}^{+}$count test and were referred to RAP during the same week. At the first RAP visit, treatment eligibility was determined (when an NHLS $\mathrm{CD}^{+}$count was not available) or confirmed via the PIMA (Alere Healthcare, Waltham, Mass, USA) point-of-care (POC) $\mathrm{CD}^{+}$machine. Women were screened for psychosocial distress (Kessler-10 scale) and alcohol misuse (Alcohol Use Disorders Identification Test (AUDIT) scale) with referrals to further care or additional RAP counsellor support during pregnancy. Eligible women $\left(\mathrm{CD} 4^{+} \mathrm{T}\right.$-cell count $<350$ cells/ $\mu \mathrm{l}$ or WHO stage III/IV) were offered ART immediately, with counselling and laboratory testing occurring on the same day. Comprehensive contact details were obtained to ensure that participants could be informed immediately of any abnormal laboratory results. Women attended the ART programme until 6 - 8 weeks after delivery, and were then transferred to standard ART services. A comparison between this model and the current standard of care is presented in Table 1 .

\section{Evaluation}

Antenatal data, including the number of patients tested and their HIV status, were extracted from on-site PMTCT registers. The cohort for analysis included all HIV-positive women who had commenced treatment through the RAP programme by the end of 2011 and were postpartum or had a final outcome available by the end of March 2012. Infant HIV diagnosis via polymerase chain reaction (PCR) testing was followed until June 2012.

Baseline information was collected from clinical records. This included demographic details, results from the psychosocial distress and alcohol misuse scales and eligibility criteria information $\left(\mathrm{CD} 4^{+}\right.$ cell count results from the PIMA POC machine and/or the NHLS together with WHO clinical staging).

Regimens and reasons for regimen changes as well as safety laboratory blood test results were collected, along with documentation of all clinical adverse events. In addition, the $\mathrm{CD} 4^{+}$ cell count and VL, feeding practices, retention in care and birth outcomes, including infant PCR results, were obtained from the patient files and recorded in a prospectively maintained database. PCR results were verified using the regional NHLS laboratory database.
Data were analysed using STATA (version 11). Bivariate analyses employed two sample $t$-tests, chi-square tests and Fisher's exact tests, as appropriate; 95\% confidence intervals (CIs) were based on the binomial distribution and all statistical tests were two-sided at $a=0.05$. Data analysis used an intention-to-treat method in which all women who were initiated onto treatment by RAP were included, regardless of delays to initiation, as this is a more conservative approach. All participants provided written informed consent, and the study was approved by the University of Cape Town Research Ethics Committee.

\section{Results}

From February 2011 until the end of December 2011, 3330 out of 3479 women who attended the ANC (95.7\%) were tested for HIV; 344 women (10.3\%) were HIV-positive, with 160 women (46.5\%) eligible for treatment according to guidelines.

Of the 160 ART-eligible pregnant women, 1 declined to participate. This analysis included 134 of these women who were postpartum or had a final outcome available by the end of the study period, and is described here.

\section{Baseline characteristics}

Table 2 describes the baseline demographic and clinical characteristics of the cohort. The mean age of the participants was 28 years, with a mean gestational age at presentation of 23 weeks (range 6 - 39 weeks). The mean $\mathrm{CD} 4{ }^{+}$cell count was 241 cells/ $\mu$ for the PIMA and 244 cells $/ \mu$ for the NHLS results. Ninety-two women (68.7\%) were diagnosed during their current pregnancy, and 37 participants (27.6\%) did not disclose their status before their first RAP visit.

\section{Baseline blood results and adverse events}

Blood samples were sent for baseline safety laboratory tests on the same day as the patients were diagnosed. On average these results were obtained 1 - 2 days later. No clinical adverse events that required medical intervention were reported. Four women had AIDS Clinical Trial Group (ACTG) grade 1 toxicity for alanine transaminase (ALT), 3 had ACTG grade 1 toxicity above the upper limit for haemoglobin, and there were no results above the upper limit for creatinine.

Table 1. Comparison of current standard of care for ART initiation in pregnancy with the new model

\begin{tabular}{l} 
Current standard of care \\
\hline HIV testing conducted on site at first antenatal care visit \\
Await return of laboratory $\mathrm{CD} 4^{+}$test results to determine treatment eligibility \\
(up to 2 weeks' delay)
\end{tabular}

Referral to separate ART services for ART-eligible pregnant women (up to 2 weeks' delay and potential for women to be lost in the referral process) Clinical work-up prior to ART commencement (up to 2 weeks' delay waiting for laboratory results)

Two-week treatment readiness/patient education programme before treatment commencement*

Home visit conducted and in some cases treatment of partner required prior to treatment commencement ( $\geq 1$ week's delay)

\section{RAP model}

Same procedure

Point-of-care $\mathrm{CD} 4{ }^{+}$count enumeration to determine treatment eligibility if laboratory tests not available - potential for same-day initiation

Integration of antenatal and ART services on site - potential for same-day initiation

Clinical work-up conducted concurrently with treatment initiation if no clinical contraindications identified. Potential for patient recall if necessary

Intensive, prioritised patient counselling the same day as treatment commencement, and then ongoing on subsequent visits No home visit conducted before treatment commencement, but contact made in the event of missed visits 


\section{Baseline regimens and regimen changes}

The most common ART regimen started was tenofovir (TDF) with lamivudine (3TC) and a non-nucleoside reverse transcriptase inhibitor, either efavirenz (EFV) (106 women (81.5\%)) or nevirapine (NVP) (13 women $(10.0 \%)$ ). Eight women $(6.2 \%)$ were commenced on TDF/3TC and ritonavir/lopinavir (LPV/r) and 1 woman each on zidovudine (AZT) and 3TC/NVP, stavudine (d4T) and 3TC/EFV, and d4T/3TC/ NVP. There were 8 regimen changes, 4 of 8 women who started ART in the first trimester changing because of LPV/r intolerance.

\section{Speed of treatment initiation}

Of the 130 women who started treatment, 118 (90.8\%) initiated ART the same day treatment eligibility was determined. Five patients (3.8\%) delayed treatment by 1 week, $3(2.3 \%)$ delayed by $2-3$ weeks, and 4 (3.1\%) delayed by 5 or more weeks. In 4 cases the delay was due to psychosocial reasons and in 6 it was for medical reasons; 5 patients in the latter group were suspected of having tuberculosis (TB). All TB suspects were negative for TB on laboratory sputum cultures, with the return time for these results (standard time of 43 days) delaying their initiation. Further details are shown in Table 3.

\section{Maternal outcomes}

Of the 134 women who were ART eligible, 4 did not commence ART for psychosocial or medical reasons (see Fig. 1 for more detail). Before delivery, 3 women were lost to follow-up (LTFU) and 4 transferred to other ART clinics. Of the 123 women who delivered during the RAP programme, 8 were LTFU before an infant PCR result was available (Fig. 1). The mean duration of treatment before delivery was 14.3 weeks (range 1 - 32 weeks). Of the 99 women who had a VL available at the time of delivery, $75 \mathrm{had}$ an undetectable VL ( $<50$ copies $/ \mathrm{ml})$. For the 24 women with a detectable VL, the median VL was 328.50 copies $/ \mathrm{ml}$ (interquartile range 118 - 16497 copies $/ \mathrm{ml}$ ).

\section{Birth outcomes}

Birth outcomes were available for 126 infants, including three sets of twins. There were 119 (94.4\%) live births, 5 miscarriages from 4 pregnancies, 2 stillbirths, 1 early neonatal death and 2 late neonatal deaths. Details of the infant deaths are presented in Table 4 . The mean ( \pm standard deviation (SD)) birth weight $(n=107)$ was $3000 \pm 580 \mathrm{~g}$ (range $1060-4600 \mathrm{~g}$ ), and 43 infants (35.2\% of the available 122 results) were born via caesarean section. Feeding choices were available for 117 of the 119 live births, with 67 mothers (57.3\%) choosing exclusive breastfeeding, 49 (41.9\%) choosing exclusive formula feeding and 1 choosing mixed feeding.

PCR results of the infants were verified in all but 13 cases (12.1\%) from laboratory databases: there were 107 negative results (from 106 pregnancies) and 1 positive result, which translates into a vertical transmission rate of $0.9 \%$ (CI $0.02-5.05 \%$ ). When the negative infant PCR results were compared with the 1 positive result and the 11 infants with unknown PCR outcomes, no significant differences in maternal baseline characteristics (age, gestational age, baseline $\mathrm{CD}^{+}$count, psychological distress, alcohol misuse, disclosure and new or known HIV status) were found.

Of the 24 women who were not virally suppressed at the time of delivery, 21 had negative PCR results, 1 had a positive infant PCR outcome (perinatal transmission rate $0.9 \%$ ), 2 had infant deaths and 2 were LTFU. The mother of the infant with the positive PCR was commenced on TDF/3TC/EFV at 35 weeks' gestation, with a baseline $\mathrm{CD}^{+}$count of 222 cells/ $\mu$ l. She was on treatment for 24 days prior to delivery, with erratic adherence. She defaulted from treatment shortly after delivery with a VL of 247 copies/ml recorded 39 days after delivery.
Table 2. Baseline characteristics of 134 ART-eligible pregnant women with a birth or final outcome available by March 2012

\begin{tabular}{ll}
\hline Characteristic & \\
Age (years), mean \pm SD (range) & \\
$\quad$ Maternal & $28.3 \pm 5.3(16$ \\
Gestational & $22.5 \pm 6.3(6-$ \\
CD4 ${ }^{+}$count (cells/ $\mu$ l), mean \pm SD (range) & \\
$\quad$ PIMA test ${ }^{*}$ & $241.1 \pm 98.9$ \\
NHLS & \\
WHO stage, $n(\%)$ & $244.5 \pm 85.6$ \\
I & \\
II & $52(38.8)$ \\
III & $48(35.8)$ \\
IV & $29(21.6)$ \\
Missing data, $n(\%)$ & $4(2.9)$ \\
Gravidity, $n(\%)$ & $1(0.7)^{\ddagger}$ \\
1 & \\
2 & $22(16.4)$ \\
$\geq 3$ & $57(42.5)$ \\
\end{tabular}

Parity, $n(\%)$

1

$\geq 2$

HIV disclosure at first RAP visit, $n(\%)$

$\begin{array}{ll}\text { Disclosed } & 97(72.4) \\ \text { Not disclosed to anyone } & 37(27.6)\end{array}$

First HIV diagnosis, $n(\%)$

HIV diagnosis in this pregnancy $92(68.7)$

Previous diagnosis $41(30.6)$

Missing data

$1(0.8)$

Psychological distress (Kessler-10 scale), $n$ (\%)

None

$110(82.1)$

Mild

Moderate

Severe

Missing data

Alcohol misuse (AUDIT scale), $n$ (\%)

$\begin{array}{ll}\text { Low risk } & 121(90.2) \\ \text { Hazardous } & 6(4.5) \\ \text { Harmful } & 2(1.5) \\ \text { Dependent } & 1(0.7) \\ \text { Missing data } & 4(3.0)\end{array}$

ART $=$ antiretroviral therapy; $\mathrm{SD}=$ standard deviation; NHLS = National Health aboratory Service; $\mathrm{WHO}=$ World Health Organization; $\mathrm{RAP}=$ Rapid Initiation in Pregnancy; AUDIT = Alcohol Use Disorders Identification Test. ${ }^{*}$ Not done in 14.

${ }^{\dagger}$ No data available for 41

†'Woman permanently deferred. 
Table 3. Details of ART initiation timing and reasons for delay $(N=130)$

\begin{tabular}{lll}
\hline Initiation speed & Frequency, $\boldsymbol{n}(\%)$ & Reason for delay in ART initiation \\
\hline First RAP visit & $118(90.8)$ & NA \\
1 week's delay & $5(3.8)$ & Medical - concurrent UTI (1) \\
& & Medical - TB suspect $(2)^{\star}$ \\
& & Psychosocial - not ready (1) \\
& & Psychosocial - wished to disclose (1) \\
2 weeks' delay & $2(1.7)$ & Mental health - depression $(1)$ \\
& & Confirmatory CD4 $4^{+}$count low $(1)^{\dagger}$ \\
3 weeks' delay & $1(0.8)$ & Medical - TB suspect $(1)^{\star}$ \\
5 or more weeks' delay & $4(3.1)$ & Medical - TB suspect $(2)^{\star}$ \\
& & Confirmatory CD4 $4^{+}$count low $(1)^{\dagger}$ \\
& & Psychosocial - requested TOP $(1)$
\end{tabular}

ART = antiretroviral therapy; RAP = Rapid Initiation in Pregnancy; NA = not applicable; UTI = urinary tract infection; TB = tuberculosis; TOP = termination of pregnancy; $\mathrm{POC}=$ point of care; NHLS = National Health Laboratory Service.

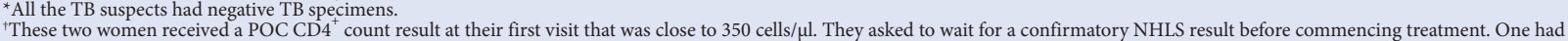
a gestational age of 11 weeks and the other 19 weeks at their first visit.

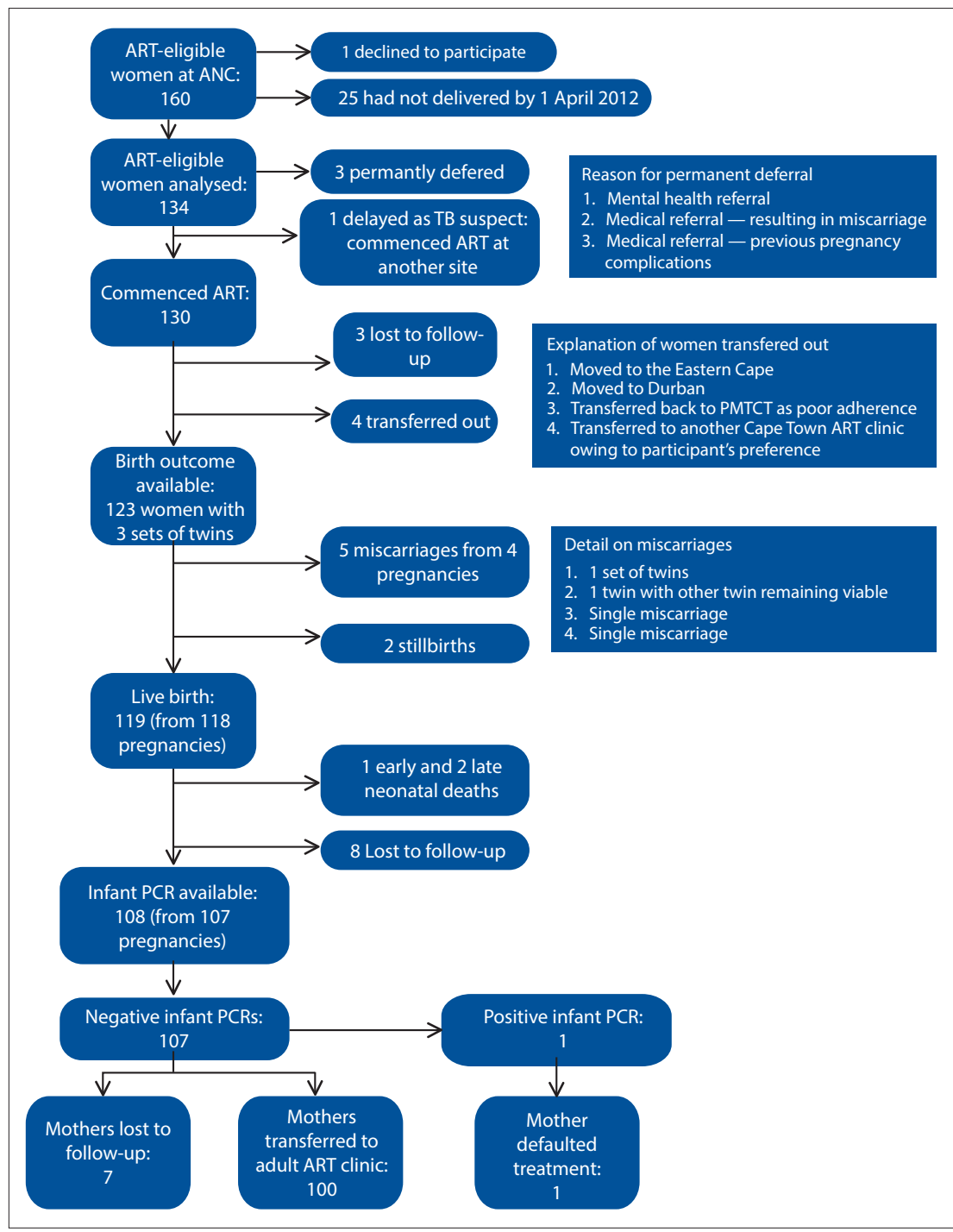

Fig. 1. Consort diagram of 160 antiretroviral therapy-eligible women who attended the Rapid Initiation in Pregnancy programme in 2011.

\section{Discussion}

This pilot study demonstrates that perinatal transmission rates can be reduced to below $1 \%$ in women with advanced HIV and relatively low $\mathrm{CD}^{+}$counts, when ART is offered as soon as eligibility is determined. POC $\mathrm{CD} 4^{+}$ testing in the ANC, concurrent collection and active monitoring of baseline bloodwork and extensive participant counselling enabled some women to commence ART on the same day as diagnosis. Furthermore, the pilot was a safe intervention and uptake in the ANC was high.

The risk of MTCT declines with increasing duration of ART. ${ }^{[7,8]}$ In SA, where women often present to health services late in pregnancy, rapid ART initiation is necessary. The RAP model changes the current SA preparation approach on two levels, with counselling and safety blood assessment occurring alongside, rather than before, ART commencement.

\section{Safety}

SA national HIV treatment guidelines recommend specific safety blood tests before commencement of treatment to evaluate the risk of toxicity. ${ }^{[16-18]}$ Serum creatinine levels are measured, and when the creatinine clearance is $<50 \mathrm{ml} / \mathrm{min}$, the patient is referred for a renal consultation with TDF alternatives recommended. In addition, ALT is measured if an NVP-based regimen is envisaged. If the ALT level is $>100 \mathrm{U} / 1$, alternatives to NVP are preferred and serological tests for hepatitis are performed ${ }^{[18]}$ Assessment of haemoglobin may be recommended, particularly if AZT is a treatment choice. This testing and return of results can delay treatment commencement. Given that treatment delays have a significant 


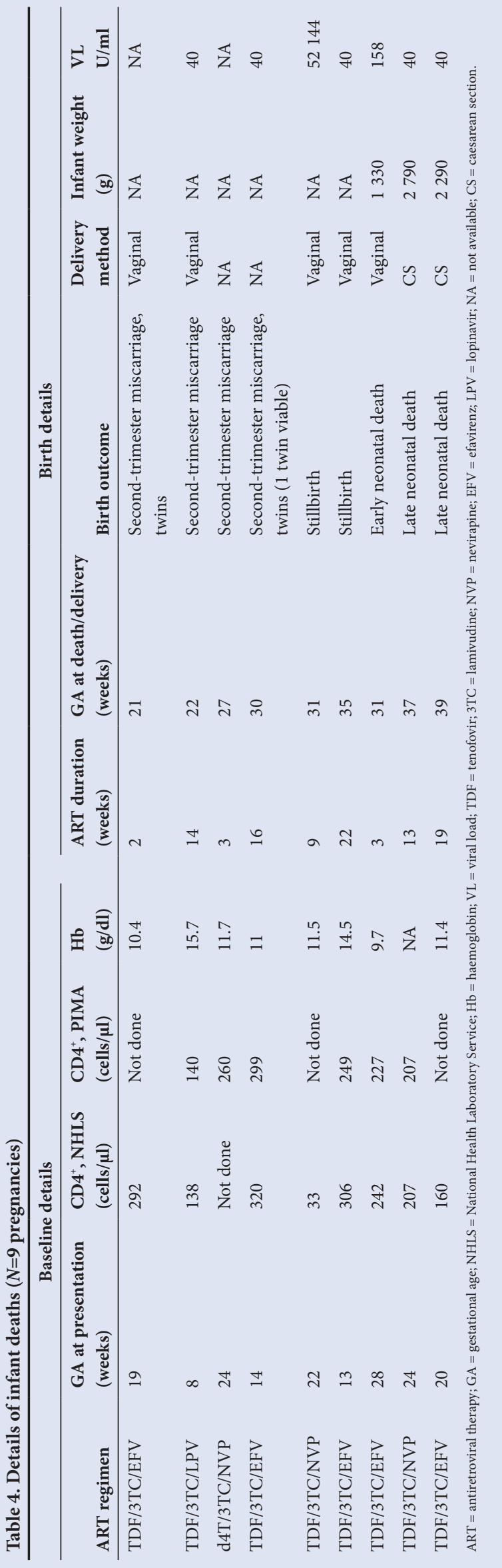

impact on vertical transmission, the relative risk of ART toxicity must be weighed against the risk of delayed viral suppression and increased perinatal transmission.

In this relatively small cohort, baseline laboratory screening tests did not identify any risk of toxicity that would have necessitated an alternative regimen or further clinical investigations. If this model is to be expanded, the results of blood tests should be available promptly and patient contact details should be in place so that modifications can be made to ART management, taking any abnormal results into account. Furthermore, in this cohort there were no clinical adverse events that required medical intervention. There were no maternal deaths, and the proportions of live births (94.4\%) and neonatal deaths (2.4\%) are comparable to those in other studies of treatment-naïve women initiating ART during pregnancy. ${ }^{[9,19]}$ The overall indications are that rapid ART initiation with concurrent screening blood tests in pregnancy is safe.

Since perinatal HIV transmission risk is greatest at the time of birth, earlier ANC booking by women would increase the likelihood of viral suppression by this time. This desirable public health outcome will require community-wide mobilisation and education. It is encouraging that rapid initiation of ART can be undertaken, particularly in those women who book late.

\section{Feasibility}

Ninety-one per cent of women were able to commence ART at their first programme visit. This represents a dramatic shift in the patient preparation procedure and highlights the feasibility of rapid initiation. Of note, $41.7 \%$ of the delays were due to the time taken for the results of TB sputum cultures to be available for women suspected of being co-infected with TB. Practitioners preferred to exclude a TB diagnosis before ART commencement. Placing POC TB screening within the ANC may reduce these delays further. ${ }^{[20]}$

In ART programmes across $\mathrm{SA}$, pregnant women are significantly more likely to be LTFU than their non-pregnant peers. ${ }^{[21]}$ In RAP, women received intensive counselling alongside, rather than before, treatment initiation. They therefore had less time to process and disclose their diagnosis, with $68.7 \%$ of the cohort receiving a new HIV diagnosis and $27.6 \%$ not having disclosed their status to anyone at the time of ART initiation. Support was bolstered by a counsellor available on call and active patient tracing following missed visits. Although RAP required additional resources for such intensive support, a separate cost-utility analysis suggests that the RAP package of services is a highly cost-effective intervention in comparison with standard services (Zulliger R, et al., 'Cost-effectiveness of a package of interventions for expedited antiretroviral therapy initiation during pregnancy in Cape Town, South Africa' - unpublished data). The RAP programme followed women until their baby's PCR was available (6 - 12 weeks). Three patients (2.3\%) were LTFU before delivery and $8(6.0 \%)$ after delivery, but before a PCR result was available. When RAP is compared with another Cape Town clinic, pre-delivery LFTU is similar. ${ }^{[9]}$ However, the cohort will need to be followed over time to see whether rapid initiation increases the risk of LTFU in this setting.

\section{Efficacy}

In RAP, the mean treatment duration prior to delivery was 14.3 weeks. This compares favourably with a retrospective analysis of standard services conducted at a neighbouring Cape Town ART clinic, which demonstrated a median duration on treatment before delivery of 7.6 weeks for ART-eligible pregnant women. ${ }^{[9]}$ Virological response was difficult to determine owing to limited VL testing in the national programme. For most women a VL was recorded at the time of delivery. These VL data demonstrated viral 
suppression for the majority of women (75.8\%), which translated to a vertical transmission rate of $0.9 \%$ (CI $0.02-5.05 \%$ ) when the unknown PCR results were excluded. A recent SA study, investigating vertical transmission rates in three provinces following the WHO 2009 guideline shift (increasing ART $\mathrm{CD}^{+}$cell count eligibility in pregnancy from $<200$ to $<350$ cells $/ \mu \mathrm{l}$ ), found a vertical transmission rate of $2.4 \%$ (CI $1.9-3.1 \%$ ) for ART-eligible women. ${ }^{[22]}$ It is probable that rapid initiation of ART in our study contributed to PMTCT rates decreasing to below $1 \%$.

Most patients were initiated by a nurse trained in ART care supervised by a doctor, illustrating that the service can be run by adequately trained midwives and suggesting that this programme is not only feasible but scalable within ANCs.

It is important to note, however, that the relatively small number of participants in the RAP pilot project limits the power to generalise on the safety and efficacy of results. The excellent reduction in transmission rates and very little evidence of toxicity support more extensive investigation of this intervention. Further limitations of this study are that it was operational, so complete data were not available for all participants; in addition, the lack of a control, standard-of-care arm means that attributing the reduction in MTCT to the rapid initiation of ART can only be presumed. Women will continue to be followed over time to determine longerterm maternal outcomes.

This pilot study demonstrates the feasibility and efficacy of rapid ART initiation in pregnancy to reduce the risk of HIV transmission from mothers to their children. Women were able to start treatment safely, with comparable maternal outcomes, live births and neonatal deaths when clinical investigations and intensive counselling occurred concurrently instead of before treatment commencement. Together with strategies that would encourage women to present earlier in pregnancy and retain them in care, this novel approach may be a critical step in the eradication of perinatally acquired HIV. Time will tell whether this rapid ART initiation in pregnancy programme also results in better outcomes for HIV-infected mothers.

Source of funding and conflict of interests. Landon Myer is supported by an International Leadership Award from the Elizabeth Glaser Paediatric AIDS Foundation. For the remaining authors, none were declared.

Acknowledgements. We thank the women, families and staff at Hanover Park ANC and the dedicated Desmond Tutu HIV Foundation Tutu-Tester team. We also thank our partners: the Cape Town Metro District Health Services, the Western Cape Provincial Department of Health, WolaNani and mothers2mothers. Funding of the staff, and of some of the operations, was derived through the Anova Health Institute and the US Agency for International Development (USAID) through The US President's Emergency Plan For AIDS Relief (PEPFAR/.
Author contributions. Study conception: LGB, DP, RW; study design: LGB, DP, LM, SB, RZ, RM, RW; study implementation: LGB, SB, RZ, RM, RT, SJ; data collection: SB, RZ, RT, SJ; data analysis: SB, RZ; writing of paper: SB, RM, LGB; review and approval of final paper: LGB, DP, LM, SB, RZ, RM, RW, SJ, RT.

\section{References}

1. World Health Organization. Use of Antiretroviral Drugs for Treating Pregnant Women and Preventing HIV Infection in Infants. Programmatic Update. Geneva: WHO, 2012. http://whqlibdoc.who.int/ hq/2012/WHO_HIV_2012.6_eng.pdf (accessed 11 May 2012).

. Statistics South Africa. Mid Year Population Estimates 2010. Pretoria, 2010. http://www.statssa.gov.za/ publications/P0302/P03022010.pdf (accessed 12 June 2012).

3. Joint United Nations Programme on HIV/AIDS. Global Report: UNAIDS Report on the Global AIDS Epidemic, 2010. Geneva: UNAIDS, 2010. http://www.unaids.org/globalreport/ documents/20101123_globalreport_full_en.pdf (accessed 4 February 2012).

4. Garcia PM, Kalish LA, Pitt J, et al. Maternal levels of plasma human immunodeficiency virus type 1 RNA and the risk of perinatal transmission. N Engl J Med 1999;341(6):394-402. [http://dx.doi. org/10.1056/NEJM199908053410602]

5. Shapiro RL, Hughes MD, Ogwu A, et al. Antiretroviral regimens in pregnancy and breast-feeding in Botswana. N Engl J Med 2010;362(24):2282-2294. [http://dx.doi.org/10.1056/NEJMoa0907736]

6. Taha TE, Kumwenda J, Cole SR, et al. Postnatal HIV-1 transmission after cessation of infant extended antiretroviral prophylaxis and effect of maternal highly active antiretroviral therapy. J Infect Dis 2009;200(10):1490-1497. [http://dx.doi.org/10.1086/644598]

7. European Collaborative Study. Time to undetectable viral load after highly active antiretroviral therapy initiation among HIV-infected pregnant women. Clin Infect Dis 2007;44:1647-1656. [http://dx.doi. org/10.1086/518284]

8. Hoffman RM, Black V, Technau K, et al. Effects of highly active antiretroviral therapy duration and Hogimen on risk for mother-to-child transmission of HIV in Johannesburg, South Africa. J Acquir
regime Immune Defic Syndr 2010;54(1):35-41. [http://dx.doi.org/10.1097/QAI.0b013e3181cf9979]

9. Fitzgerald FC, Bekker LG, Kaplan R, Myer L, Lawn SD, Wood R. Mother-to-child transmission of HIV in a community-based antiretroviral clinic in South Africa. S Afr Med J 2010;100(12):827-831.

10. Stringer EM, Ekouevi DK, Coetzee D, et al. Coverage of nevirapine-based services to prevent motherto-child HIV transmission in 4 African countries. JAMA 2010;304(3):293-302. [http://dx.doi. org/10.1001/jama.2010.990]

11. Stinson K, Boulle A, Coetzee D, Abrams EJ, Myer L. Initiation of highly active antiretroviral therapy among pregnant women in Cape Town, South Africa. Trop Med Int Health 2010;15(7):825-832. [http://dx.doi.org/10.1111/j.1365-3156.2010.02538.x]

12. Abrams EJ, Myer L, Rosenfield A, El-Sadr WM. Prevention of mother-to-child transmission services as a gateway to family-based human immunodeficiency virus care and treatment in resource-limited settings: Rationale and international experiences. Am J Obstet Gynecol 2007;197(3 suppl):S101-106. settings: Rationale and international experien
[http://dx.doi.org/10.1016/.ajog.2007.03.068]

13. Gebrekristos HT, Milsana, KP, Karim QA. Patients' readiness to start highly active antiretorviral treatment for HIV. BMJ 2005;331(7519):772-775. [http://dx.doi.org/10.1136/bmj.331.7519.772]
to 3 .

14. Myer L, Zulliger R, Pienaar, D. Diversity of patient preparation activities before initiation of antiretroviral therapy in Cape Town, South Africa. Trop Med Int Health 2012;17(8):972-977. [http:// dx.10.1111/j.1365-3156.2012.03033

15. Myer L, Zulliger R, Black S, Pienaar D, Bekker LG. Pilot programme for the rapid initiation of antiretroviral therapy in pregnancy in Cape Town, South Africa. AIDS Care 2012;24(8):986-992. [http://dx.doi.org/10.1080/09540121.2012.668173]

16. National Department of Health South Africa. Clinical Guidelines for the Management of HIV and AIDS in Adolescents and Adults. Pretoria, 2010. http://www.who.int/hiv/pub/guidelines/south_ africa_art.pdf (accessed 12 June 2012).

17. Cooper RD, Wiebe N, Smith N, Keiser P, Naicker S, Tonelli M. Systematic review and meta-analysis: Renal safety of tenofovir disoproxil fumarate in HIV-infected patients. Clin Infect Dis 2010;51(5):496505. [https://dx.doi.org/10.1086/655681]

18. Dieterich DD, Robinson PA, Love J, O'Stern J. Drug induced liver injury associated with the use of nonnucleoside reverse transcriptase inhibitors. Clin Infect Dis 2004;38(suppl 2):S80-89. [https://dx.doi. org/10.1086/381450]

19. Black V, Hoffman R, Sugar C, et al. Safety and efficacy of initiating highly active antiretroviral therapy . Black V, Hoffman R, Sugar C, et al. Safety and efficacy of initiating highly active antiretroviral therapy
in an integrated antenatal and HIV clinic in Johannesburg, South Africa. J Acquir Immune Defic Syndr 2008;49(3):276-281. [https://dx.doi.org/10.1097/QAI.0b013e318189a769]

20. Boehme CC, Nicol MP, Nabeta P, et al. Feasibility, diagnostic accuracy, and effectiveness of decentralised use of the Xpert MTB/RIF test for diagnosis of tuberculosis and multidrug resistance: A multicentre implementation study. Lancet 2011;377(9776):1495-1505. [https://dx.doi.org/10.1016/ S0140-6736(11)60438-8]

21. Kaplan R, Orrell C, Zwane E, Bekker LG, Wood R. Loss to follow-up and mortality among pregnant women referred to a community clinic for antiretroviral treatment. AIDS 2008;22(13):1679-1681. [https://dx.doi.org/10.1097/QAD.0b013e32830ebcee]

22. Grimwood A, Fatti G, Mothibi E, Eley B, Jackson D. Progress of preventing mother-to-child transmission of HIV at primary healthcare facilities and district hospitals in three South African provinces. S Afr Med J 2012;102(2):81-83.

Accepted 11 June 2013. 